\title{
Reduction of Phenolindo-2,6-dichlorophenol in Dark and Light by the Blue-Green Alga, Anabaena variabilis
}

\author{
BY N. G. CARR AND MARY HALLAWAY \\ Department of Biochemistry, University of Liverpool
}

(Received 9 October 1964)

\begin{abstract}
SUMMARY
The reduction of phenolindo-2,6-dichlorophenol (PICP) by the biuegreen alga Anabaena variabilis was examined in the dark and in the light. The two reductions differed in order with respect to PICP, in $\mathrm{pH}$ optimum, response to cyanide and iodoacetate, and in sensitivity to starvation and lysozyme treatment of the organism. The reduction in the dark was suppressed at light intensities of $300 \mathrm{ft}$.-candles. These results are discussed in relation to work on the photo-inhibition of respiratory processes in this and other micro-organisms.
\end{abstract}

\section{INTRODUCTION}

The blue-green algae (Cyanophyceae) are structurally the simplest of the algae. Electron microscope studies have shown their resemblance to the bacteria (Ris \& Singh, 1961; Wildon \& Mercer, 1963) in that they lack morphologically distinct organelles such as nuclei, mitochondria and chloroplasts. The main reason for their inclusion among the algae is their plant-like, rather than bacterial, type of photosynthesis; they possess chlorophyll $a$ rather than bacteriochlorophyll and use water as the ultimate reductant. The Hill reaction, the photo-stimulation of $\mathrm{O}_{2}$ production on the addition of artificial oxidants, occurs not only with chloroplasts but also with intact green algae (Clendenning \& Ehrmantraut, 1950) and blue-green algae (Thomas \& De Rover, 1955). While studying the physiology of intact Anabaena variabilis we examined spectrophotometrically the reduction of a typical Hill oxidant, phenolindo-2,6-dichlorophenol by this alga, in the light and in the dark, and have attempted to characterize the two types of reaction. Recent workers (Fredricks \& Jagendorf, 1964; Krogmann \& Susor, 1964) studied Hill reactions catalysed by chlorophyll-containing fragments of blue-green algae but with particular reference to the role of phycocyanin. Fredricks \& Jagendorf (1964) noted a dark reduction of 2,3,6-trichlorophenolindophenol by chlorophyll-containing fragments; however, they suggested that this was probably due to the presence of endogenous reducing compounds. A preliminary account of some of the experiments reported has been published (Carr \& Hallaway, 1964).

\section{METHODS}

Organisms. Anabaena variabilis (Kützing) was kindly supplied by Professor J. Myers (Department of Botany and Zoology, University of Texas, U.S.A.) and Chlorogloea fritschii (Mitra) and Chlorella pyrenoidosa by Professor G. E. Fogg 
(Department of Botany, Westfield College, London), Nostoc muscorum (1453/12) and Anabaena cylindrica (1403/2) were obtained from the Culture Collection of Algae and Protozoa, Department of Botany, University of Cambridge.

Chemicals. Phenolindo-2,6-dichlorophenol (PICP) and lysozyme hydrochloride were bought from British Drug Houses Ltd. (Poole, Dorset) and 3-(3,4-dichlorophenyl)-1,1-dimethyl urea (DCMU) from K. and K. Laboratories, Inc., New York.

Medium and growth conditions. All organisms were grown on Medium C (Kratz \& Myers, 1954) supplemented with $0.05 \%(\mathrm{w} / \mathrm{v}) \mathrm{NaHCO}_{3}$. This provided an essentially inorganic medium, a small amount of sodium citrate $(0.165 \mathrm{~g} . / 1$.$) being the only$ organic constituent of medium $\mathrm{C}$; carbon dioxide was the source of carbon for growth. Stock cultures were maintained on agar $(1 \%)$ slopes of this medium and cultures for experimental purposes were grown at 32-34 ${ }^{\circ}$ in $500 \mathrm{ml}$. medium in 21 . penicillin pots, illuminated by $60 \mathrm{~W}$. daylight strip lights at a distance of 9 in., gassed with a mixture of air $+\mathrm{CO}_{2}(95+5$, by vol.) and gently shaken. Some earlier cultures were gassed with a mixture of $\mathrm{N}_{2}+\mathrm{CO}_{2}(95+5$, by vol.). The behaviour of algae with respect to PICP reduction when grown in either gas mixture was the same. Under the conditions described Anabaena variabilis grew to a population density of equiv. $0 \cdot 5-1 \cdot 0 \mathrm{mg}$. dry wt. $/ \mathrm{ml}$. in 4-6 days. Algae were harvested at room temperature by centrifugation at $500 \mathrm{~g}$ for $4 \mathrm{~min}$. washed in half their initial volume of water, recentrifuged and suspended in water to equiv. $20 \mathrm{mg}$. dry wt./ml. Growth was measured turbidimetrically, and a calibration curve used to relate EEL colorimeter reading to $\mathrm{mg}$. dry wt. $/ \mathrm{ml}$.

Measurement of reduction of oxidants. The reduction of oxidants in the light and in the dark was measured spectrophotometrically in a Unicam S.P. 700 recording spectrophotometer. A glass cuvette, fitted with a ground glass stopper, containing equiv. $1 \cdot 5-3.0 \mathrm{mg}$. dry wt. algae, oxidant to give an initial extinction of $0 \cdot 8-1 \cdot 4$ (e.g. $0.005 \%$ PICP), $\mathrm{KH}_{2} \mathrm{PO}_{4}+\mathrm{K}_{2} \mathrm{HPO}_{4}$ buffer ( $\mathrm{pH} 6.0$ ) final concentration of $0.025 \mathrm{M}$, in a total volume of $2.75 \mathrm{ml}$. was illuminated with frequent agitation at a distance of $15 \mathrm{~cm}$. from a $100 \mathrm{~W}$. tungsten bulb yielding a light intensity of about $300 \mathrm{ft}$.-c. at the cuvette and a temperature of $27-30^{\circ}$. The rate of reduction of oxidant was measured at the absorption maximum of its oxidized form (606 $\mathrm{m} \mu$ at pH 6.0 for PICP) against a blank containing algae + buffer only. The rate of reduction in the dark was measured under the same conditions but with exclusion of light; the time for the measurement of extinction in the spectrophotometer did not exceed 20 sec. Since reduced PICP is oxidized by air, in some experiments the reactions were done under nitrogen by using a modified Thunberg top to the cuvette.

Production of light of (a) known spectral region and $(b)$ known intensity. (a) When light of a particular spectral region was required the experimental cuvette was shielded from laboratory light and illuminated by using appropriate filters (see Results). Data on the transmission characteristics for the filters were obtained from Kodak Wratten Filters, 2nd edn, 1961, and from Chance-Pilkington Optical Works, St Asaph, Flint.

(b) Illumination of different intensities was obtained by using lamps of different wattage (25-300) or by interposing one or more wire-gauze screens between a $100 \mathrm{~W}$ bulb and the cuvette. In each case the intensity of incident light on the cuvette was measured by a Multi-Lux exposure meter.

Absorption spectra of pigments of Anabaena variabilis. Photosynthetic pigments 
were separated into a (chlorophyll + carotenoid-bearing) sedimentable fraction and a soluble phycocyanin-containing fraction. The algae were sonically disintegrated in a M.S.E. $60 \mathrm{~W}$. disintegrator for $3 \mathrm{~min}$. and then centrifuged at 50,000 $\mathrm{g}$ for $90 \mathrm{~min}$. The phycocyanin was obtained in the clear blue supernatant fluid and chlorophyll and carotenoids were sedimented in the pellet. The latter pigments were extracted from the pellet by a mixture of acetone + methanol $(7+2$, by vol. $)$ and their spectra recorded by a Unicam SP. 800.

\section{RESULTS}

The reduction of oxidants by microalgae

The reduction of various oxidants by different microalgae was examined under the standard conditions. Phenolindo-2,6-dichlorophenol (PICP) was reduced in the light and dark by all the algae investigated (Anabaena variabilis, A. cylindrica, Nostoc muscorum, Chlorogloea fritschii and Chlorella pyrenoidosa); in each case light

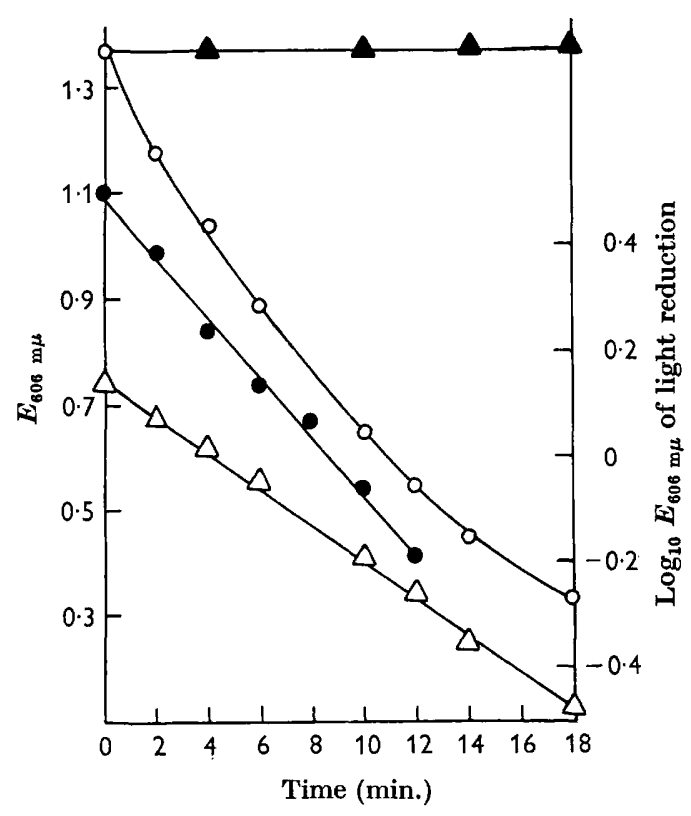

Fig. 1. Reaction course of PICP reduction by Anabaena variabilis in light and dark. Reactions measured under the standard conditions with equiv. $1.8 \mathrm{mg}$. dry wt. algae

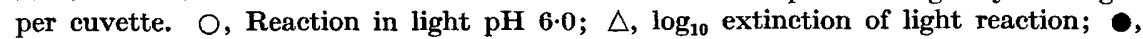
reaction in dark $\mathrm{pH} \mathrm{6.5;} \Delta$, reaction in dark and light in presence of growth media, after removal of algae. $k$ for light reaction $=7 \cdot 4 \times 10^{-4} \mathrm{sec}^{-1} / \mathrm{mg}$. dry wt. algae.

appeared either to accelerate a light-independent reduction, or to initiate a kinetically different reaction. Methylene blue was reduced, in the presence and absence of light, by $N$. muscorum and $A$. variabilis but not by $A$. cylindrica. Ferricyanide was not reduced by $A$. variabilis, either at $\mathrm{pH} 6$ or $7, \pm 0.35 \mathrm{mM}-\mathrm{MgCl}_{2}$ nor by Chlorella (Clendenning \& Ehrmantraut, 1950), although it is utilized as a Hill oxidant by isolated chloroplasts. Although benzoquinone was reduced by $A$. variabilis it is unsuitable for use with intact algae since it is known to damage them rapidly and irreversibly (Clendenning \& Ehrmantraut, 1950). The reduction of PICP by $A$. variabilis was selected for further examination. 


\section{Kinetics of the reduction of PICP in dark and light by Anabaena variabilis}

The rate of PICP reduction by Anabaena variabilis was linear with time in dark but decreased apparently exponentially in light; the corresponding logarithmic plot for the reduction in light was linear (Fig. 1). This suggested that the reduction in light was of first order and that in dark was of zero order with respect to PICP concentration. This was confirmed by the results obtained when the PICP concentration was varied in light and dark. A threefold increase in PICP concentration did not alter the rate of reduction in dark but increased proportionately the rate in light (Fig. $2 a$ ). In dark and in light the rate of reduction was directly proportional to the
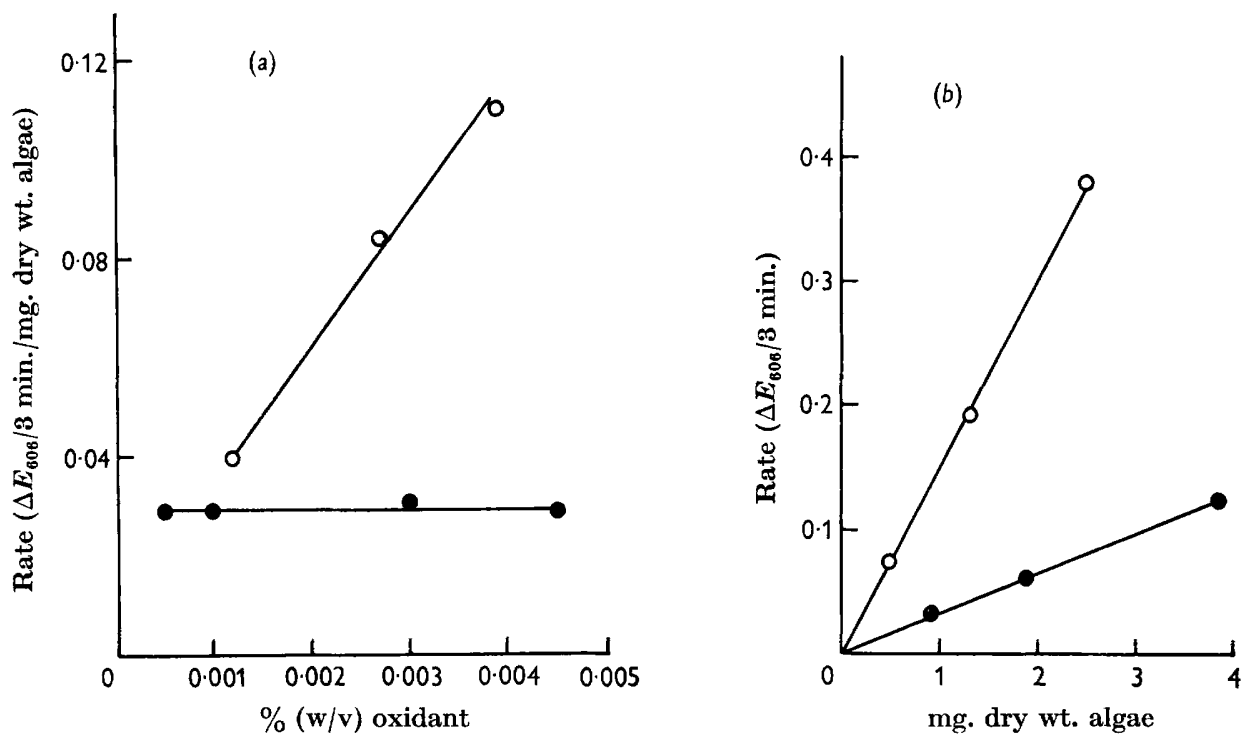

Fig. 2. Effect of (a) PICP concentration, $(b)$ algal concentration on rate of PICP reduction. $O$, Reduction in the light; $O$, reduction in the dark. Measured under the standard conditions except for the variable studied.

concentration of organism (Fig. $2 b$ ). The question arises, does the dark reduction continue in light or is it wholly or partially suppressed? The available evidence supports the hypothesis that at intensities of $300 \mathrm{ft}$.-c. and greater, light completely suppressed the dark reduction. Under the standard light conditions the rate of reduction decreased exponentially with time. When the decrease in extinction was corrected for a postulated simultaneous light-independent reduction the corrected changes fitted no standard equation of reaction kinetics. Furthermore, when the organisms were allowed to reduce part of the PICP in light and were then transferred to dark, only a slow rather erratic reduction took place (Fig. 3). In contrast, transferring the system to light after a period of reduction in dark merely induced the expected sharp change in the kinetics of the reaction (Fig. 3); the rate constant for light reaction was unaffected by the preliminary reduction in dark.

However, suppression of the dark reduction by light depended on the light intensity and the ratio of algae to PICP in the cuvettes. At a light intensity of $20 \mathrm{ft}$.-c. the reaction was indistinguishable from that in the dark; at $70 \mathrm{ft}$.-c. the light 
reaction was first order but a subsequent dark reduction was only partially suppressed. The mechanism of the suppression by light is not known; illumination of the organisms, the PICP, or both, separately, before transferring to the dark and mixing, had no inhibitory effect on the reduction which then took place in the absence of light.

None of the reducing activity of Anabaena variabilis is released into the medium, since neither the growth medium from which the organisms had been harvested, nor water in which they had been suspended had any detectable effect on PICP in dark or light (Fig. 1.).

\section{Effect of $p H$ value on PICP reduction}

The form and optimum of the effect of $\mathrm{pH}$ value on the rate of reduction in light and in dark differed (Fig. 4); the $\mathrm{pH}$ optimum of the dark reduction was $6 \cdot 5$, that of the light $6 \cdot 0$.

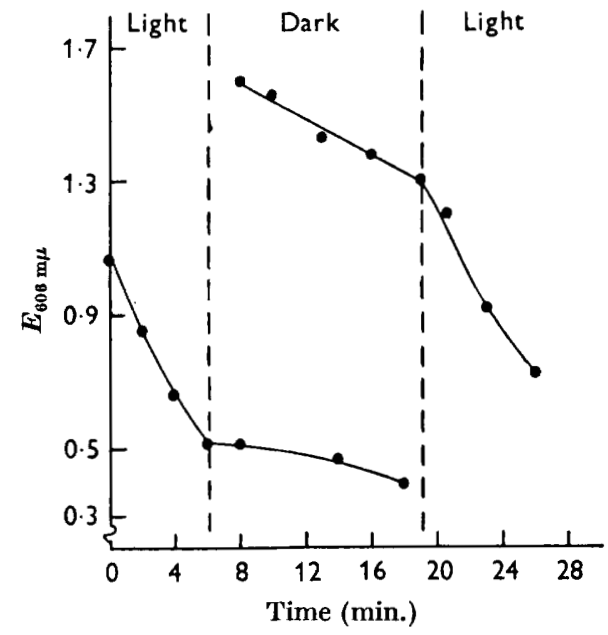

Fig. 3

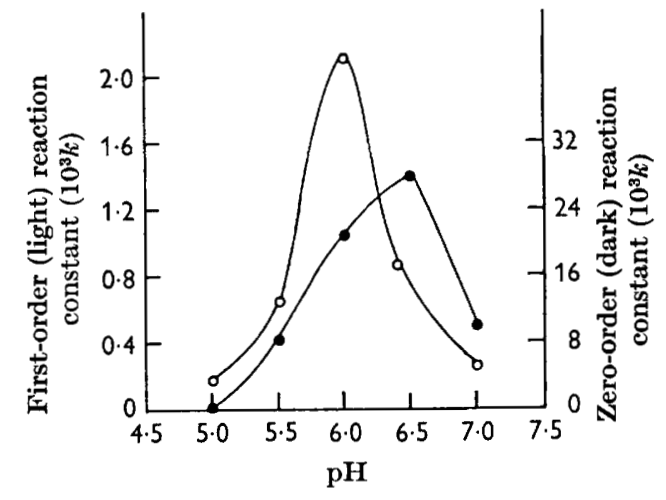

Fig. 4

Fig. 3. Alternate effects of light and dark on PICP reduction by Anabaena variabilis. Reactions carried out under the standard conditions.

Fig. 4. Effect on pH value on light and dark reduction of PICP by Anabaena variabilis. $O$, Reduction in light; $\odot$, reduction in dark. Reactions measured under the standard conditions except for the differences in $\mathrm{pH}$ value.

\section{Effect of light intensity and temperature}

The rate of the light reaction at two temperature ranges and at different intensities of light is shown in Fig. 5. Under the standard conditions (temperature 27-30 ${ }^{\circ}$ ) the rate of reduction increased with increasing light intensity over the range 45 $300 \mathrm{ft}$.-c. Increasing the temperature to $35-36^{\circ}$ increased the reduction rate at these light intensities, which suggests that light was not the sole limiting factor (Fig. 5). However, at the higher temperature, increasing the light intensity from $150 \mathrm{ft}$.-c. to $300 \mathrm{ft}$.-c. had relatively little affect on the reduction rate, so that under these conditions the light intensity was no longer rate limiting. 


\section{Utilization of light of different spectral regions}

By using different light sources in association with appropriate filters Anabaena variabilis was exposed to light of various wavelengths; the regions selected were those of maximum absorption of the $A$. variabilis pigments (Fig. 6). It was not possible with the apparatus available to adjust the light source so that the algae were illuminated with light of different wavelengths but of equal intensity, hence quantitative comparison is impossible. However PICP reduction was increased, relative to reduction in the dark, by light of each spectral region used. This suggests that light absorbed by all three pigment types was effective in promoting PICP reduction.

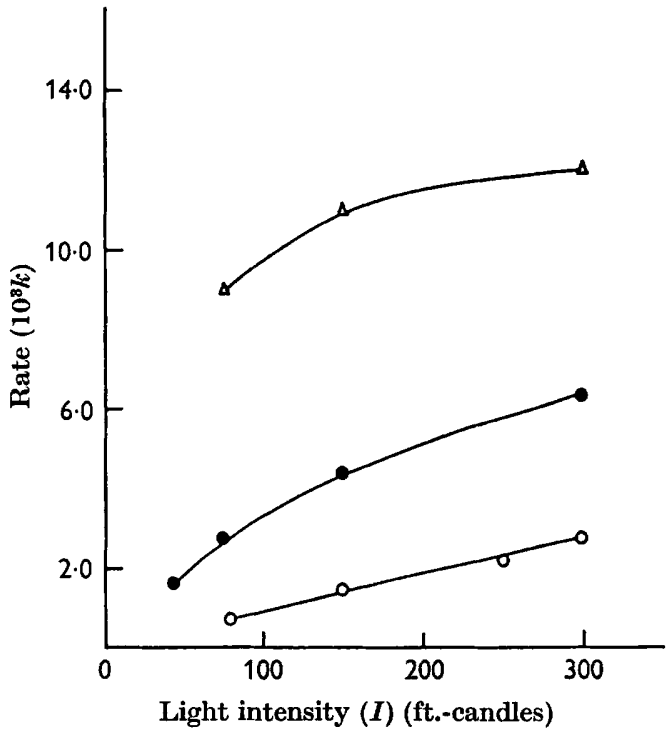

Fig. 5

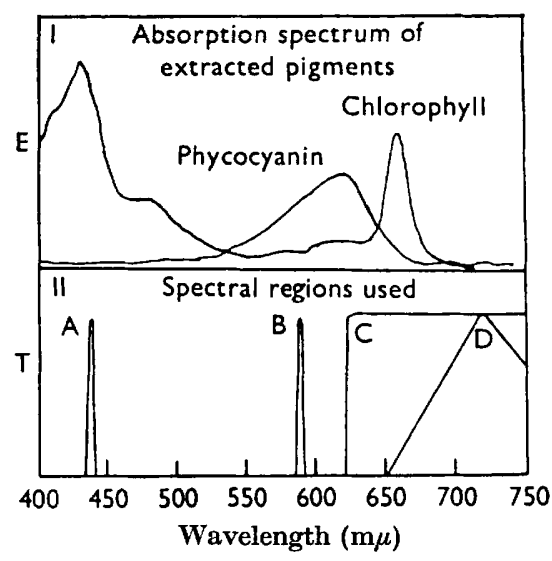

Fig. 6

Fig. 5. Effect of light intensity, at two temperatures on PICP reduction by Anabaena variabilis. The reactions were done under the standard conditions except that light intensity $(I)$ was varied by two means. $O, I$ varied by using lamps of different wattage; temperature $27-30^{\circ}$;,$I$ varied by using a $100 \mathrm{~W}$ lamp and a series of wire-gauze screens; temperature $27-30^{\circ} ; \triangle, I$ varied with wire-gauze screen; temperature $35-36^{\circ}$.

Fig. 6. (I) Absorption spectra of pigments of Anabaena variabilis and (II) spectral regions used in PICP reduction. (I) shows the absorption spectrum of the pigments extracted from Anabaena variabilis. The absorption below $500 \mathrm{~m} \mu$ is attributable to carotenoids and chlorophyll a, the peak near $600 \mathrm{~m} \mu$ to phycocyanin and the peak in the $660 \mathrm{~m} \mu$ region to chlorophyll $a$. (II) is a diagrammatic representation of the transmission characteristics of four combinations of filters and light sources devised to provide light of spectral regions absorbed by Anabaena pigments.

$\begin{array}{cl}\text { Spectral region } & \text { Light source } \\ \text { A } & \text { Mercury lamp } \\ \text { B } & \text { Sodium lamp } \\ \text { C } & \text { Mercury lamp } \\ \text { D } & \text { Tungsten lamp }\end{array}$

Filter

Kodak Wratten 98

None

Kodak Wratten 29

Kodak Wratten 29, Chance OX 7 


\section{Effect of inhibitors}

The difference between light and dark reaction kinetics, in $\mathrm{pH}$ optimum, and the existence of a light inhibition of the dark reduction, all indicate that the reduction reactions in light and dark differed in mechanism and presumably also in the source of hydrogen used in the reaction. This possibility was examined by testing the effect of various compounds on the light and dark reductions. The reactions differed in their response to cyanide $(0.5-1 \cdot 0 \mathrm{~mm})$ and iodoacetate $(0 \cdot 7-1 \cdot 4 \mathrm{mM})$ both of which stimulated the dark reduction but did not affect the light reduction. Since in the dark PICP is probably acting as an alternative electron acceptor to oxygen stimulation by cyanide, which inhibits cytochrome oxidase is not unexpected; stimulation by iodoacetate is not easy to explain since if it were inhibiting glycolysis this would retard the flow of electrons to PICP. Neither $0.7 \mathrm{~mm}$-hydroxylamine hydrochloride nor 0.1 mu 3-(3,4-dichlorophenyl)-1,1-dimethyl urea (DCMU) inhibited the reduction in light; both are inhibitors of the oxygen producing stages of photosynthesis but DCMU does not inhibit photoreductions (Gingras, Lemasson \& Fork, 1964; Hoch, Owens \& Kok, 1963).

\section{Reduction of PICP by starved Anabaena variabilis}

The oxygen consumption and PICP reduction by organisms that had been starved of $\mathrm{CO}_{2}$ by gassing with $\mathrm{CO}_{2}$-free air for $5 \mathrm{hr}$ before harvesting were examined and compared with organisms from the same culture which had been gassed with $5 \%$ $(\mathrm{v} / \mathrm{v}) \mathrm{CO}_{2}+95 \%(\mathrm{v} / \mathrm{v})$ air until harvested. Starvation did not alter the rate of the light reaction significantly, but the dark reaction was no longer linear and ceased after about half the PICP had been reduced. The endogenous respiration of the starved algae was less than $50 \%$ of that obtained with normal organisms. These observations accord with the suggestion that the endogenous reserves are the source of the electrons for the dark reduction, but not for the reaction in light.

\section{Table 1. Effect of lysozyme treatment of Anabaena variabilis on PICP reduction}

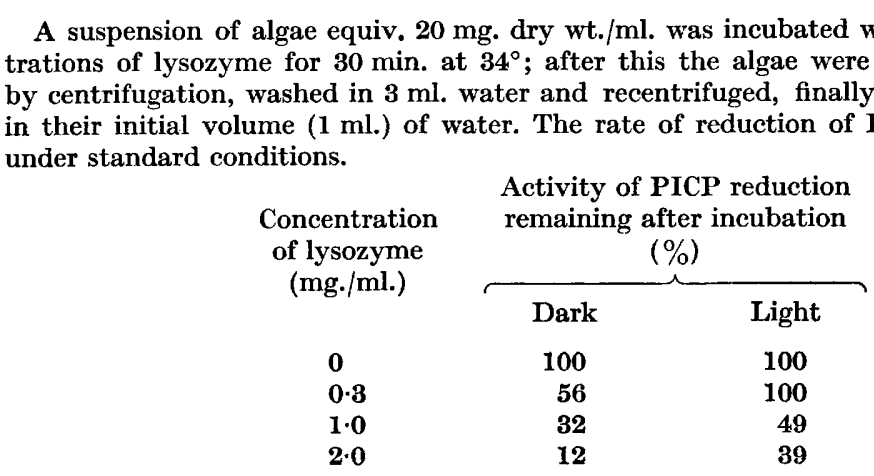

\section{Reduction of PICP by Anabaena variabilis treated with lysozyme}

Incubation of a washed suspension of algae with lysozyme resulted in release of phycocyanin and a rapid decrease in the rate of both dark and light reduction of PICP. The reduction in dark appeared to be the more sensitive to lysozyme treat- 
ment. The decrease in rate of the reaction in dark and light after $30 \mathrm{~min}$. incubation with different concentrations of lysozyme is shown in Table 1. Addition of phycocyanin to inactivated preparations did not restore the ability to reduce PICP.

If the PICP was acting as final electron acceptor additional to oxygen it might be expected that lysozyme treatment which retarded the transfer of electrons to PICP would also retard the respiratory transfer of electrons to oxygen. However, oxygen uptake due to endogenous respiration of Anabaena variabilis was not decreased by incubation with lysozyme, even when $75 \%$ of the phycocyanin had been lost and PICP reduction in light and dark was barely detectable (Fig. 7). The initial loss of

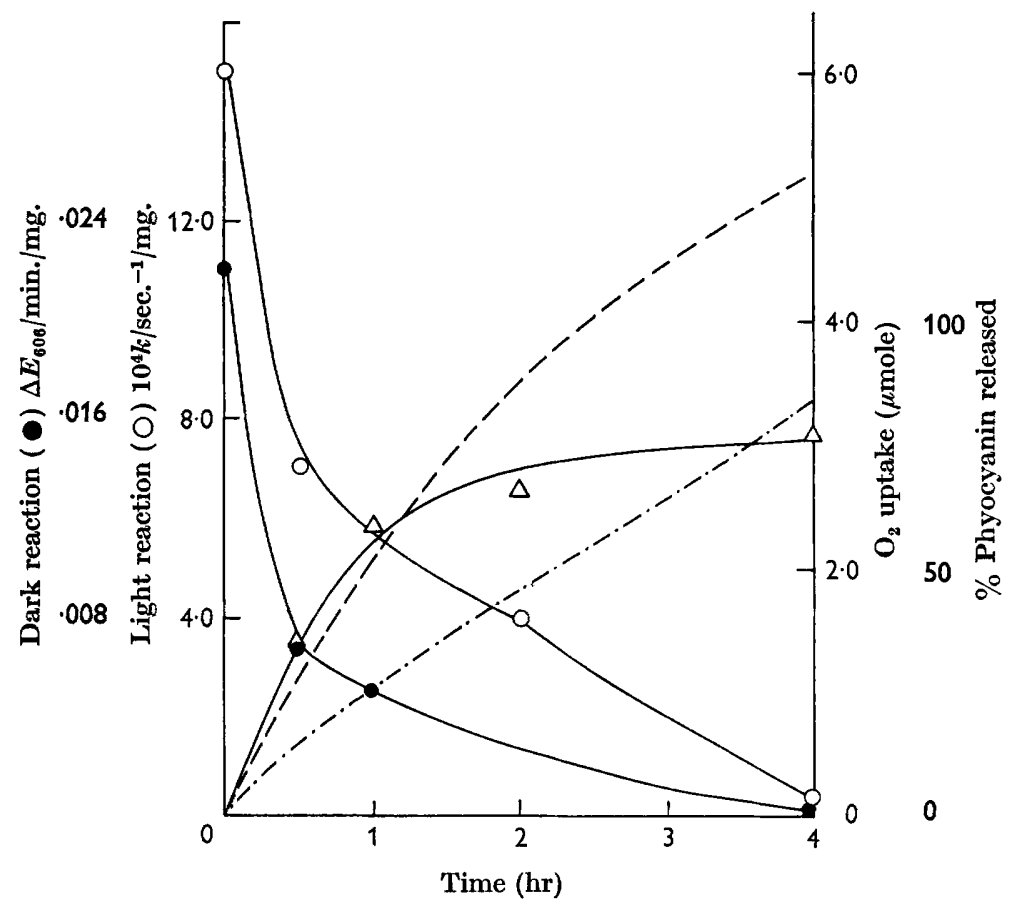

Fig. 7. Effect of lysozyme treatment on release of phycocyanin, respiration and light and dark reduction of PICP by Anabaena variabilis. O, PICP reduction in light; 9, PICP reduction in dark; -...-, endogenous respiration in the presence of lysozyme; ......, endogenous respiration without lysozyme treatment; $\Delta$, \% phycocyanin released. The PICP reductions were measured on lysozyme-treated algae under the standard conditions. Endogenous respiration was measured by $\mathrm{O}_{2}$ uptake by the direct manometric method. The release of phycocyanin into the supernatant fluid by lysozyme treatment was estimated by measuring the extinction at $610 \mathrm{~m} \mu\left(E_{610}\right)$ and comparison with the extinction of the total phycocyanin obtained by sonic disruption of the algae followed by centrifugation at 50,000 $\mathrm{g}$ for $90 \mathrm{~min}$.

phycocyanin from the algae into the medium was rapid; over $50 \%$ of the total phycocyanin was released during the first hour of incubation, coinciding with the marked decrease in ability to reduce PICP. There was no indication that the lysozyme treatment had lysed the organisms, since they could still be sedimented by centrifuging at $500 \mathrm{~g}$ for $\mathbf{5}$ min., and appeared intact by microscopic examination. 


\section{DISCUSSION}

Phenolindo-2,6-dichlorophenol (PICP) has often been used as an artificial oxidant in studying both respiratory and photosynthetic electron transfer. With an $E_{0}^{\prime}$ of $+295 \mathrm{mV}$. at $\mathrm{pH} \mathrm{6.0} \mathrm{it} \mathrm{would} \mathrm{be} \mathrm{reduced} \mathrm{by} \mathrm{flavoproteins} \mathrm{and} \mathrm{could} \mathrm{be} \mathrm{oxidized} \mathrm{by}$ some cytochromes of the respiratory chain. The point at which PICP may be reduced in photosynthetic electron transport is still not clear (Gromet-Elhanan \& Avron, 1964). Our results show that Anabaena variabilis can reduce PICP in the dark and light, that the two reductions differ, and that light suppresses the dark reaction. It seems probable that the ultimate reductant in the light is water, and in the dark is the endogenous substrates of the organism, but the mechanisms of the two reductions and of the light suppression of the dark reaction are not yet established.

Suppression of respiratory oxygen uptake by light has been noted for both bluegreen algae (Brown \& Webster, 1953, Hoch, Owens \& Kok, 1963) and purple bacteria (van Niel, 1941; Fork \& Goedheer, 1964). To explain the phenomenon Brown \& Webster (1953) suggested that either the photosynthetically produced oxygen was utilized within the organism (an explanation which cannot hold for the bacteria in which oxygen is not a product of photosynthesis), or that light inhibited respiratory electron transfer. Assuming that PICP is acting in both presence and absence of light merely as an electron acceptor, then our results support the latter suggestion.

The interaction of the light and dark reduction of PICP by Anabaena variabilis could be another example of the structural and functional linkage of photosynthesis and respiration found in procaryotic organisms and discussed by Stanier \& van Niel (1962). Procaryotic organisms, bacteria and blue-green algae, differ from eucaryotic organisms in that their genetic material is not enclosed by a defined membrane separating it from the cytoplasm nor do they possess membrane bound organelles specialized for different functions, e.g. chloroplasts for photosynthesis or mitochondria for respiration. Hence the electron transport chains of procaryotic organisms may serve both in respiration and photosynthesis so that photosynthetic electron transport may exclude respiratory electron transfer (see Horio \& Kamen, 1962).

We wish to thank Dr R. Wayne for the loan of light sources and filters and for his advice on their use. This work was supported in part by a research grant AM-05282-03 from the U.S. Public Health Service.

\section{REFERENCES}

Brown, A. H. \& Webster, G. C. (1953). The influence of light on the rate of respiration of the blue-green alga Anabaena. Am. J. Bot. 40, 753.

Carr, N. G. \& Hallaway, M. (1964). Phenolindo-2,6-dichlorophenol reduction by Anabaena variabilis (Kützing). Biochem. J. 91, 15 P.

Clendenning, K. A. \& Ehrmantraut, H. C. (1950). Photosynthesis and Hill reactions by whole Chorella cells in continuous and flashing light. Arch. Biochem. Biophys. 29, 387.

Fork, D. C. \& GoedheER, J. C. (1964). Studies on light induced inhibition of respiration in purple bacteria: action spectra for Rhodospirillum rubrum and Rhodopseudomonas spheroides. Biochim. biophys. Acta. 79, 249.

Fredricks, W. W. \& J AgEndorf, A. T. (1964). A soluble component of the Hill reaction in Anacystis nidulans. Arch. Biochem. Biophys. 104, 39. 
Ginfras, G., Lemasson, C. \& Fonk, D. C. (1963). A study of the mode of action of 3-(4-dichlorophenyl)-1,1-dimethyl urea (CMU) on photosynthesis. Biochim. biophys. Acta, 69, 438.

Gromet-Elhanan, Z. \& Avron, M. (1964). The role of indophenol dyes in photoreactions of chloroplasts. Biochemistry, 3, 365.

Hoch, G., Owens, O. V. H. \& KoK, B. (1963). Photosynthesis and respiration. Arch. Biochem. Biophys. 101, 171.

Horio, T. \& KAmen, M. D. (1962). Observations on the respiratory system of Rhodospirillum rubrum. Biochemistry, 1, 1141.

Kratz, W. A. \& MYers, J. (1954). Nutrition and growth of several blue-green algae. Am. J. Bot. 42, 282.

Krogmann, D. W. \& Susor, W. A. (1964). Hill reaction in cell free preparations of a bluegreen alga. Fed. Proc. 23, 226.

VAN Niel, C. B. (1941). The bacterial photosyntheses and their importance for the general problem of photosynthesis. Advanc. Enzymol. 1, 263.

Ris, H. \& Singh, R. N. (1961). Electron microscope studies on blue-green algae. J. biophys. biochem. Cytol. 9, 63.

Stanier, R. Y. \& van Niel, C. B. (1962). The concept of a bacterium. Arch. Mikrobiol. $42,17$.

Thomas, J. B. \& De Rover, W. (1955). On phycocyanin participation in the Hill reaction of the blue-green alga Synechococcus cedrorum. Biochim. biophys. Acta, 16, 391.

Wildon, D. C. \& Mercer, F. V. (1963). The ultrastructure of the vegetative cell of bluegreen algae. Aust. J. biol. Sci. 16, 585. 